\title{
Designing anti-sliding durable type of asphalt mixture based on the maximum peak of angular distribution rate
}

\author{
Duan yi Wang, Yong tan Yu, Gang Wang ${ }^{\mathrm{a}}$, Chi Xu and Shen shen Shao \\ School of Civil Engineering and Transportation, South China University of Technology, Guangzhou 510641, China
}

\begin{abstract}
In order to design a anti-sliding and durable asphalt mixture, we independently develop two sets of laboratory equipment: the Laser Contour Detector of Asphalt Pavement and the Accelerated Rub Tester, with which we collect the plate surface contour every two hours for 5 times in the first 8 hours in the rub test, and then we make contour curves and analyze the angular distribution statistics of surface contour and thus we propose characterizing the skid resistance performance and durability of the asphalt pavement based on the maximum peak of angular distribution rate, which has been proved to be reasonable. By designing different gradations of SMA-13 and GAC-13 asphalt mixture and using the maximum peak of angular distribution rate to evaluate the skid resistance performance of different degrees of kneading, the asphalt mixture with better skid resistance has been made. That provides a reference for engineering application. The laboratory research develops a method for designing a anti-sliding and durable asphalt mixture based on the maximum peak of angular distribution rate, which makes up for the defects of the current stage where there is no ways to test the skid resistance performance and durability while designing the asphalt mixture.
\end{abstract}

\section{Introduction}

Most Chinese highways use asphalt pavement whose skid resistance performance is directly related to the safety of road traffic, and the skid resistance performance is an important index of road function, therefore the study on the skid resistance performance of asphalt pavement is very important. The problem of the skid resistance performance of asphalt pavement is serious, especially the rapid attenuation of the skid resistance durability. There are many factors that can influence the skid resistance durability, among them the asphalt mixture gradation design and pavement construction technology have the bigger impacts [1 5]. The average texture depth of the road would decay rapidly from $1.0 \mathrm{~mm}$ to $0.6 \mathrm{~mm}$ and the cornering ratio would also decay rapidly from $60 \sim 70$ to $40 \sim 50$ after carrying traffic for two years[6 7]. All those are not accord with the requirements of trunk road traffic safety and disobey the industry requirements. In order to solve those problems, this thesis developed two sets of experimental equipment Laser Contour Detector and Accelerated Rub Tester. Focusing on the factors like different degrees of roughness and different kneading time, we use the road profile peak angle to study the skid resistance and durability of the asphalt pavement[8 11], aiming to establish related quantitative evaluation way to evaluate the skid resistance performance and durability of asphalt mixture and also attempting to put forward new indexes to design the asphalt pavement with good skid resistance and durability.

\footnotetext{
${ }^{a}$ Corresponding author : $1873620714 @ q q . c o m$
} 
In order to adapt to the current unceasing skid resistance development situation of the big structure pavement, this thesis used statistics of the macro profile peak angle to study the macroscopic outline of asphalt pavement. Focusing on the roughness of different road surface and different knead time and combining with the asphalt pavement laser detector and acceleration load knead machine, it also established relevant quantitative evaluation method and attempted to put forward new indexes to evaluate skid resistance durability of the asphalt pavement.

\section{Objective and scope of this study}

(1) Two sets of independent research and development laboratory equipment: First: Asphalt Pavement Laser contour detector, Second: Accelerated rub tester.

(2) Proposed maximum peak angular distribution rate to characterize the asphalt pavement It skids durability. using the angular distribution of the maximum peak rate evaluation of skid resistance rub their different stages.

(3) Rub in the room combined with accelerated loading test machine, laser contour detector, forming a peak with maximum angular distribution rate Durable skid design of asphalt mixture method, make up this stage of asphalt mixture design phase does not validate its durability against sliding of defects.

\section{Laboratory tests and data collection}

\subsection{Working principle introduction of the laser contour detector}

The self-developed laser contour detector in our research is made up of three parts: measurement system, control system and data acquisition system. It could control measurement points within the measurement range of the asphalt pavement surface and store measuring data into computer in real time. It is as shown in figure $1 \sim 3$.

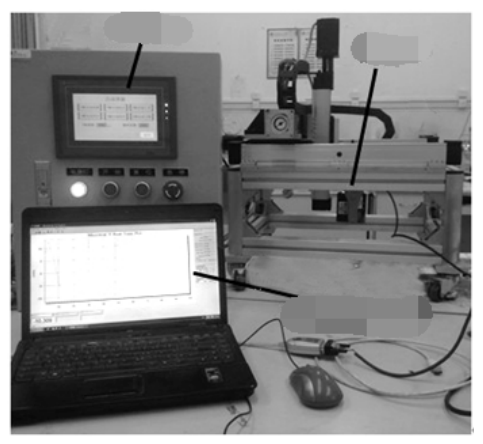

Figure 1. Laser contour detector.

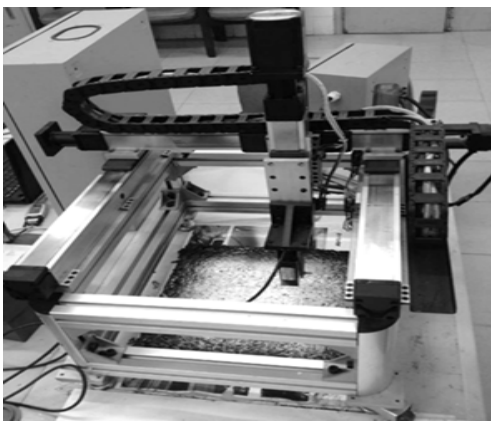

Figure 2. Laser contour detection plate specimen measured rut.

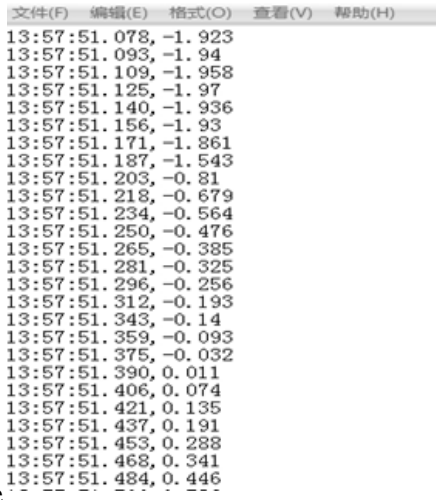

Figure 3. Measurement data.

\subsection{Working principle introduction of the acceleration load kneading machine}

When the tester is working, the driving screw steel bar will make the specimen platform in horizontal movement repeatedly with the effect of the new motor while the old motor will go on driving the traction wheels in vertical movement, so the tire could make back and forth movement on the test rut board. Besides, the thermostatic water bath equipment is added and its adjustable temperature range is from indoor temperature to $70^{\circ} \mathrm{C}$. The lateral movement of the apparatus is $10 \mathrm{~cm} / \mathrm{min}$; The wheels travel $42 \pm 1$ times per minute. The wheel weighs $42-100 \mathrm{~kg}$ (adjustable). At the same time, considering the real tire rolling on the road has the thread, we make thread on the smooth tire in order to imitate 
the actual driving wheel. It is shown as figure 4-a. This tester can be operated simply and fast and the simulation environment is effective.

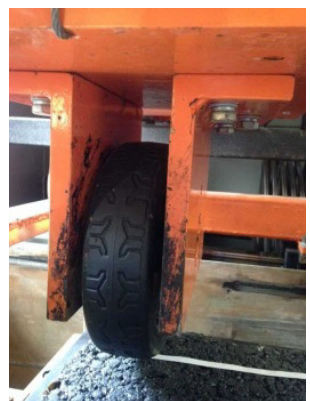

(a)

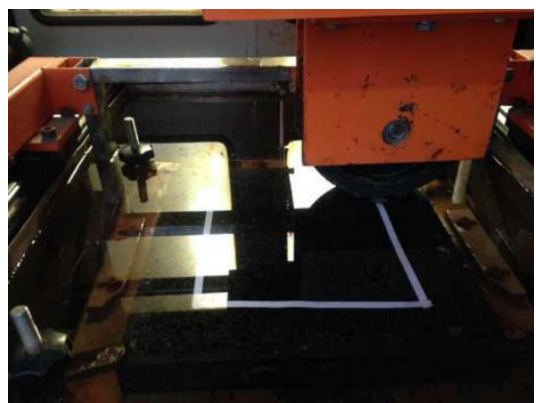

(b)

Figure 4. Kneading machine and working principle.

In this research process, we use the self-developed accelerated rub tester as well the laser contour detector, and improve the gradation composition of AC-13 and its design method based on CAVF method and broken material gradation design theory. So we get the asphalt mixture whose skid resistance surface gradation is of discontinuous $4.75 \mathrm{~mm}$ sieve holes. For distinguishing from the traditional standard grading, we call the AC-13 of discontinuous gradation $(4.75 \mathrm{~mm})$ the skeleton dense type gradation GAC-13(Gap-graded Asphalt Concrete) whose grading curve is as shown in table 1. We compare the dense-suspended type of AC-13 with this kind of GAC-13, using two kinds of rut boards made by these two kinds of asphalt mixture. The size of these rut boards is $300 \times 300 \times 50 \mathrm{~mm}$ which has $100 \mathrm{~kg}$ wheel load. After kneading the rut boards of GAC- 13 and $\mathrm{AC}-13$ under $60^{\circ} \mathrm{C}$ water environment in $0,2,4,6,8$ hours, we dry out the rut boards and test them with the laser contour detector.

Table 1. Gradation of two asphalt samples (\%)

\begin{tabular}{ccccccccccc}
\hline \multirow{2}{*}{ Mixture } & \multicolumn{1}{c}{ Sieve (Square Hole Sieve $\boldsymbol{m m}$ ) } \\
& 16 & 13.2 & 9.5 & 4.75 & 2.36 & 1.18 & 0.6 & 0.3 & 0.15 & 0.075 \\
\hline AC-13 & 100 & 95 & 78.5 & 51.5 & 35 & 25.5 & 18 & 13.5 & 10 & 6 \\
GAC-13 & 100 & 95.2 & 62.5 & 26.9 & 22.1 & 16.4 & 12.1 & 9.2 & 7.3 & 6.3 \\
\hline
\end{tabular}

To show their difference of roughness, we use the self-developed 3-D laser texture measuring equipment to simulate 3-D diagram of the pavement of the AC-13 and GAC-13, which is shown as figure 6. We also figure out the MTD of the rut board of the AC-13 and GAC-13 is $1.36 \mathrm{~mm}$ and $0.85 \mathrm{~mm}$ respectively. We use indoor sanding method to measure the initial skid resistance performance of these two rut boards molded indoor. The results show that texture depth of GAC-13 rut board is $1.2 \mathrm{~mm}$, while that of $\mathrm{AC}-13$ is $0.92 \mathrm{~mm}$. Measured by tilting friction pendulum tester, the initial BPN of GAC-13 rut board is 78 , while that of $\mathrm{AC}-13$ is 70 . It is quite clear that the initial skid resistance performance of GAC-13 rut board is better than AC-13 rut board. Therefore, we choose these two kinds of rut boards which have significant differences on initial texture depth and BPN as the simulation road in the experiment.

\subsection{Principle of the profile peak angle calculation}

The profile curve of the rut board surface could be got after denoising and filtering process of the date collected by the laser contour detector. Then, statistical analysis is carried out on peak angle value of the contour curve. The calculation of peak angle value $\alpha$ is as shown in figure 5 and formula (1): 


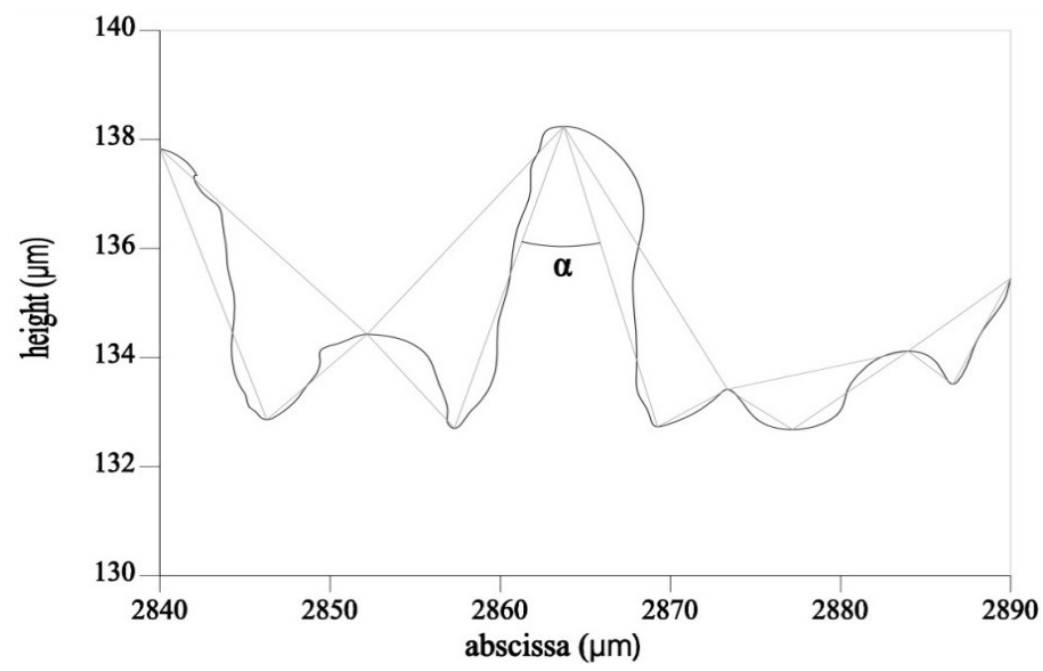

Figure 5. Schematic of outline peak angle.

$$
\alpha=\tan ^{-1}\left|\frac{x_{i}-x_{i-1}}{y_{i}-y_{i-1}}\right|+\tan ^{-1}\left|\frac{x_{i+1}-x_{i}}{y_{i+1}-y_{i}}\right|, i=1,2,3 \ldots \mathrm{n}
$$

In this formula: $\mathrm{x}_{\mathrm{i}}$

is the value of the No. $i$ extreme point on $\mathrm{x}$ axis. When the No. $i$ point reaches to the summit, the No. $i-1$ point and the No. $i+1$ point are at the bottom; $\mathrm{y}_{\mathrm{i}}$ is the value of No. $i$ extreme point on $\mathrm{y}$ axis.

Each wave crest of the contour curve could be considered as an aggregate particle on the pavement, while the profile peak angle value represent the form of prominent aggregate particles. The smaller the angle value is, the narrower the aggregate particles are, while the bigger, the wider.

\subsection{Data collection}

After importing the measured data of laser contour detector into the MATLAB computer programs, we could got a TXT file (figure 7) by the statistical analysis. The specific operation is as shown in figure 6 .

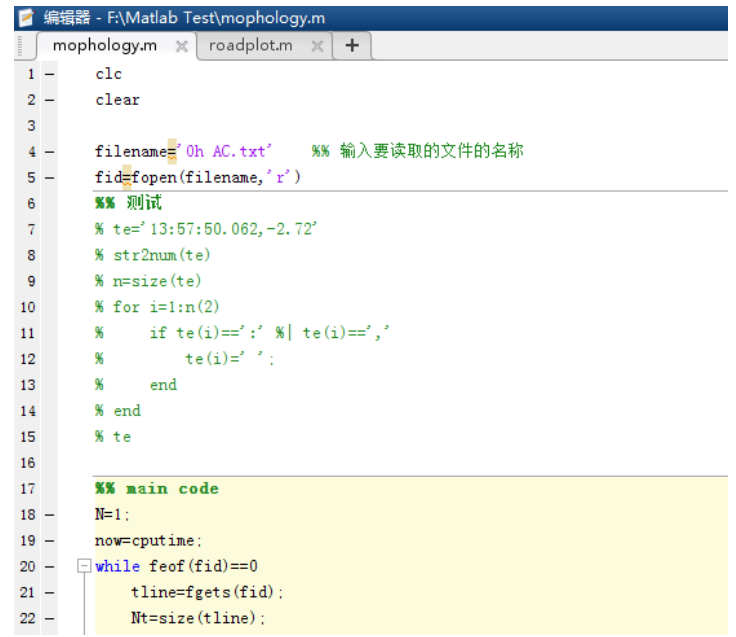

Figure 6. MATLAB data processing.

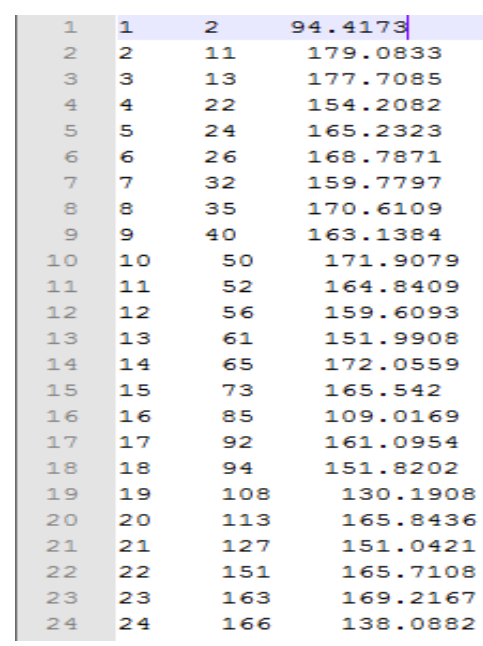

Figure 7. Data output format. 
According to the measurement scheme and test method introduced above, we measure the profile of the dried rut boards with laser contour detector after knead tests in every two hours, namely, in $0,2,4,6,8$ hours and map the profile curve and calculate the angle $\alpha$. The profile peak angle directly determines the macro profile of rut boards. In every test area, there are 1000 test lines and each of them has thousands of peaks, so in every test of each rut board there are thousands values of $\alpha$. In this paper, the number of the profile peak angles in every 10 degrees are counted, which means there are 18 sections: $0-10^{\circ}, 10-20^{\circ}, \ldots, 170-180^{\circ}$. Every probability distribution in each area is calculated to illustrate the distributed situation of profile angles. The statistics of the surface profile angles of these two rut boards are as shown in figure 10 and 11. We take the largest peak angle range of $150 \sim 180$ degrees as the analysis object of this study, and define it as the maximum peak of angle distribution rate. Table 2 is the rate of maximum peak angular distribution and the final rate of change.

\subsection{Asphalt Mixture gradation design}

Generally, the anti-sliding performance of SMA-13 and GAC-13 asphalt mixture with skeleton dense structure in the above layer is better than AC-13 asphalt mixture with dense-suspended structure. In this section, we use SMA-13 and GAC-13 gradation as the research object, trying to design the asphalt mixtures with different skeleton structure and evaluate the anti-sliding performance and durability of them with maximum peak of angular distribution rate so as to to select the best asphalt mixture of anti-sliding layer. In the paper written by Liwan $\mathrm{Shi}^{[12]}$, through the research of contact force of the backbone chain in SMA-13 mixture, he found that the aggregate under $2.36 \mathrm{~mm}$ almost didn't participate in the composition of main chain. Therefore, in the test of this section, we choose two kinds of aggregate of SMA-13 mixture which are $9.5 \mathrm{~mm}$ and $4.75 \mathrm{~mm}$ to study the best anti-sliding gradation. Considering GAC-13 has the same skeleton dense structure, we design it in the same way, and use AC-13 asphalt mixture with dense-suspended structure as comparison study.

The raw material of asphalt mixture is made by diabase and SBS modified asphalt mentioned in the former test. All the specimen is selected with the nominal maximum particle size of $13.2 \mathrm{~mm}$; the SMA mineral powder dosage is $10.0 \%$ while that of GAC-13 and AC mixture is $4 \%$ respectively. SMA is made from lignin fiber, with $0.3 \%$ dosage of the mixture and mixed within the aggregate. The asphalt dosage of SMA is $6.0 \%$, while that of GAC-13 and AC-13 is $4.8 \%$ each. According to the above design points, 3 pieces of rut board with each kind of gradation are made. We conduct knead test in $0,2,4,6,8$ hours, carry out laser test in different kneading phase and write computer program MATLAB to make statistic analysis of maximum peak angular distribution rate. We use the average value of these three parallel tests as the final analysis object. The pass percentage of different gradation in different level is shown in table $2 \sim 3$.

Table 2. SMA-13 quality pass percentage of different graduation in different level.

\begin{tabular}{c|c|c|c|c|c|c|c|c|c|c}
\hline \multirow{2}{*}{$\begin{array}{c}\text { Grading } \\
\text { type }\end{array}$} & \multicolumn{10}{|c}{ Sieve (Square Hole Sieve $\mathbf{m m}$ ) (\%) } \\
\cline { 2 - 11 } & 16 & 13.2 & 9.5 & 4.75 & 2.36 & 1.18 & 0.6 & 0.3 & 0.15 & 0.075 \\
\hline SMA-1 & 100 & 95.0 & 70.0 & 35.0 & 22.5 & 19.0 & 16.5 & 14.0 & 12.8 & 10.0 \\
\hline SMA-2 & 100 & 95.0 & 65.0 & 35.0 & 22.5 & 19.0 & 16.5 & 14.0 & 12.8 & 10.0 \\
\hline SMA-3 & 100 & 95.0 & 60.0 & 35.0 & 22.5 & 19.0 & 16.5 & 14.0 & 12.8 & 10.0 \\
\hline SMA-4 & 100 & 95.0 & 60.0 & 30.0 & 22.5 & 19.0 & 16.5 & 14.0 & 12.8 & 10.0 \\
\hline SMA-5 & 100 & 95.0 & 60.0 & 25.0 & 22.5 & 19.0 & 16.5 & 14.0 & 12.8 & 10.0 \\
\hline SMA-6 & 100 & 95.0 & 55.0 & 25.0 & 22.5 & 19.0 & 16.5 & 14.0 & 12.8 & 10.0 \\
\hline SMA-7 & 100 & 95.0 & 50.0 & 25.0 & 22.5 & 19.0 & 16.5 & 14.0 & 12.8 & 10.0 \\
\hline AC & 100 & 95.0 & 78.5 & 51.5 & 35.0 & 25.5 & 18.0 & 13.5 & 10.0 & 6.0 \\
\hline
\end{tabular}

Table 3. GAC-13 quality pass percentage of different graduation in different level.

\begin{tabular}{c|c|c|c|c|c|c|c|c|c|c}
\hline \multirow{2}{*}{$\begin{array}{c}\text { Grading } \\
\text { type }\end{array}$} & \multicolumn{10}{|c}{ Sieve (Square Hole Sieve $\mathbf{m m}$ ) (\%) } \\
\cline { 2 - 11 } & 16 & 13.2 & 9.5 & 4.75 & 2.36 & 1.18 & 0.6 & 0.3 & 0.15 & 0.075 \\
\hline GAC-1 & 100 & 95.2 & 70.0 & 35.0 & 22.1 & 16.4 & 12.1 & 9.2 & 7.3 & 6.3 \\
\hline
\end{tabular}




\begin{tabular}{c|c|c|c|c|c|c|c|c|c|c}
\hline GAC -2 & 100 & 95.2 & 65.0 & 35.0 & 22.1 & 16.4 & 12.1 & 9.2 & 7.3 & 6.3 \\
\hline GAC -3 & 100 & 95.2 & 60.0 & 35.0 & 22.1 & 16.4 & 12.1 & 9.2 & 7.3 & 6.3 \\
\hline GAC -4 & 100 & 95.2 & 60.0 & 30.0 & 22.1 & 16.4 & 12.1 & 9.2 & 7.3 & 6.3 \\
\hline GAC -5 & 100 & 95.2 & 60.0 & 25.0 & 22.1 & 16.4 & 12.1 & 9.2 & 7.3 & 6.3 \\
\hline GAC -6 & 100 & 95.2 & 55.0 & 25.0 & 22.1 & 16.4 & 12.1 & 9.2 & 7.3 & 6.3 \\
\hline GAC -7 & 100 & 95.2 & 50.0 & 25.0 & 22.1 & 16.4 & 12.1 & 9.2 & 7.3 & 6.3 \\
\hline AC & 100 & 95.0 & 78.5 & 51.5 & 35.0 & 25.5 & 18.0 & 13.5 & 10.0 & 6.0 \\
\hline
\end{tabular}

\section{Test results and discussion}

\subsection{Skid resistance durability evaluation of the asphalt pavement based on the macro profile peak angle}

The following table 4 is maximum peak of angle distribution rate and the final rate of change after different kneading time.

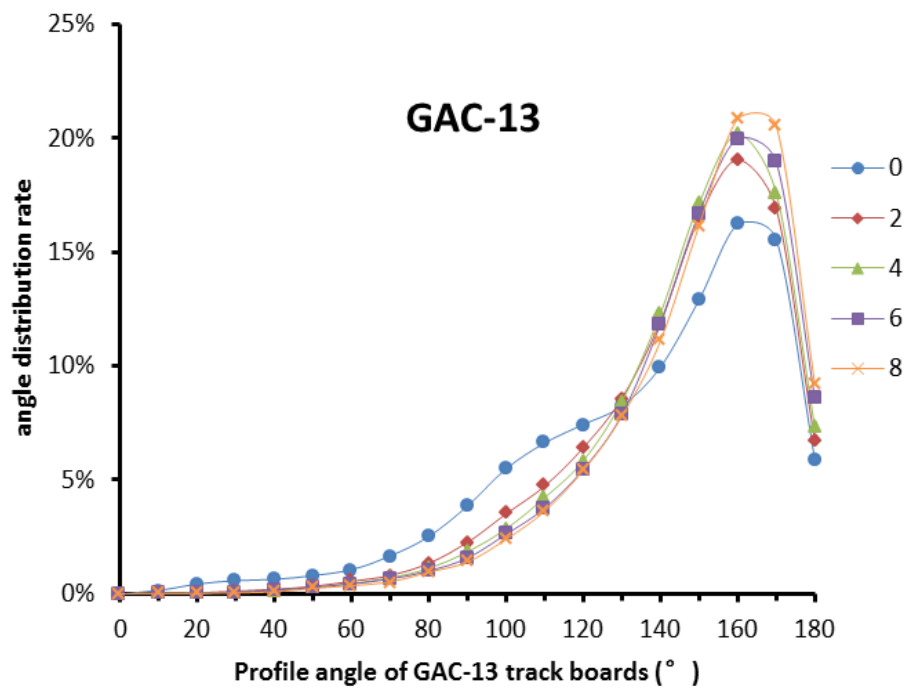

Figure 8. GAC-13 Road surface contour angle distribution chart.

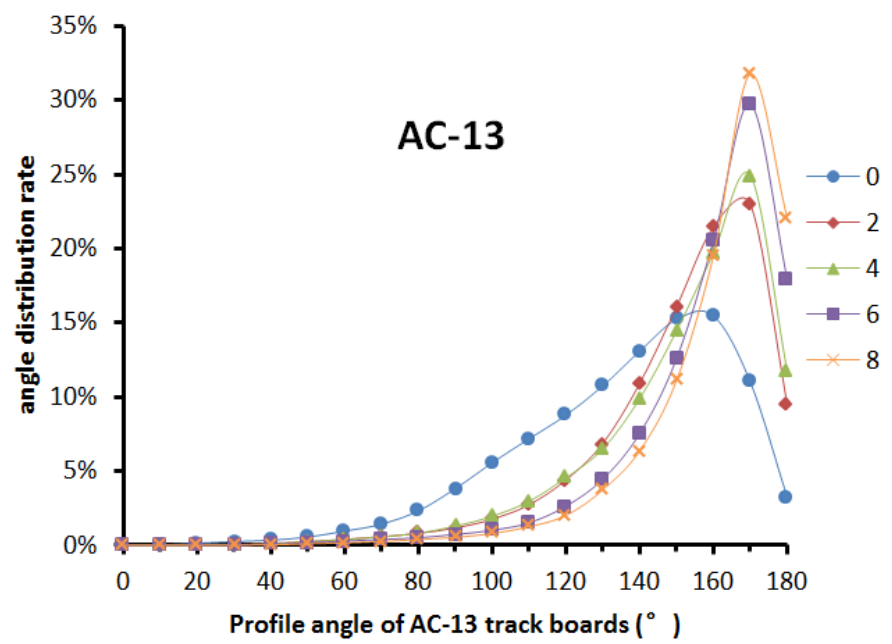

Figure 9. AC-13 Road surface contour angle distribution chart. 
Table 4. Rate of maximum peak angle distribution and the final rate of change.

\begin{tabular}{|c|c|c|c|c|c|c|c|}
\hline $\begin{array}{c}\text { Grading } \\
\text { Type }\end{array}$ & $\begin{array}{c}\text { Evaluation } \\
\text { Index }\end{array}$ & Oh & $2 \mathrm{~h}$ & $4 \mathrm{~h}$ & $6 \mathrm{~h}$ & $8 \mathrm{~h}$ & \multirow{2}{*}{$\begin{array}{c}\text { Rate of } \\
\text { change } \\
(\%)\end{array}$} \\
\hline \multirow{2}{*}{ AC-13 } & Angle range & $150-180$ & $150-180$ & $150-180$ & $150-180$ & $150-180$ & \\
\hline & rate $(\%)$ & $54.9 \%$ & $70.0 \%$ & $70.8 \%$ & $80.8 \%$ & $84.4 \%$ & $34.98 \%$ \\
\hline \multirow{2}{*}{ GAC-13 } & Angle range & $150-180$ & $150-180$ & 150-180 & $150-180$ & $150-180$ & \multirow{2}{*}{$23.43 \%$} \\
\hline & rate $(\%)$ & $50.6 \%$ & $59.2 \%$ & $61.7 \%$ & $64.2 \%$ & $66.0 \%$ & \\
\hline
\end{tabular}

After kneading the rut boards of AC-13 and GAC-13 in 0, 2, 4, 6, 8 hours, we dried them under the normal temperature and used sanding method and tilting friction pendulum tester to measure the MTD and BPN of the two rut boards whose variation trends are as shown in figure 10 to 11 , and the correlation analysis is shown in figure 12.
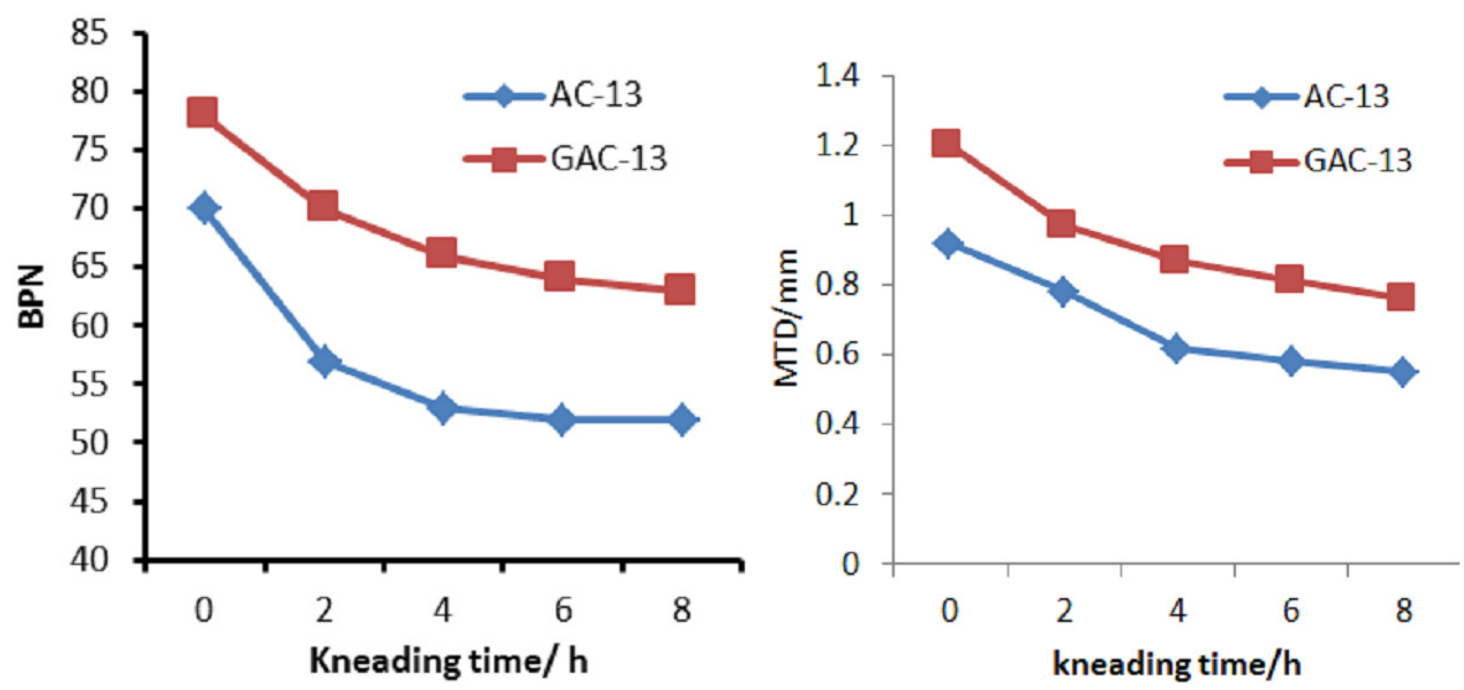

Figure 10. Texture Depth trends.

Figure 11. Pendulum value trends.

As we can see from figure 8 and figure 9, the analysis of profile peak angle value $\alpha$ shows good unimodality which is an obvious peak, and the profile peak angle value is basically in line with the gamma distribution. The result shows that the acute angle change is not obvious and the peak angle distribution ratio is small, and the obtuse angle would increase with kneading time going by, which means that the pavement profile angle is getting flat and its skid resistance performance is declining. Therefore, the skid resistance performance attenuation degree of GAC-13 is lower than that of AC-13. Besides, the initial and final skid resistance performance of GAC-13 is better than that of AC-13. In this contrast test, the MTD and BPN of the two rut boards are fading, which is to say the skid resistance performance is declining. The correlation between maximum peak angle distribution rate and BPN as well as MTD of GAC-13 is very good, and $\mathrm{R}^{2}$ has achieved 0.99 . The correlation between maximum peak angle distribution rate and BPN as well as MTD of AC-13 is good , and $\mathrm{R}^{2}$ has exceeded 0.84. so it is a verification to the proposal that we can use maximum peak of angle distribution rate to represent pavement skid resistance performance. In summary, it's reasonable and accurate to use the maximum peak angle distribution rate to represent the skid resistance performance and durability of the pavement. 


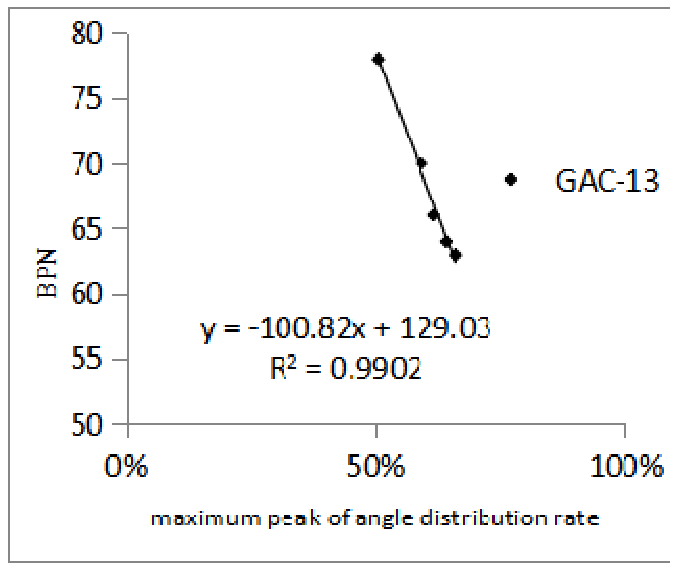

(a)

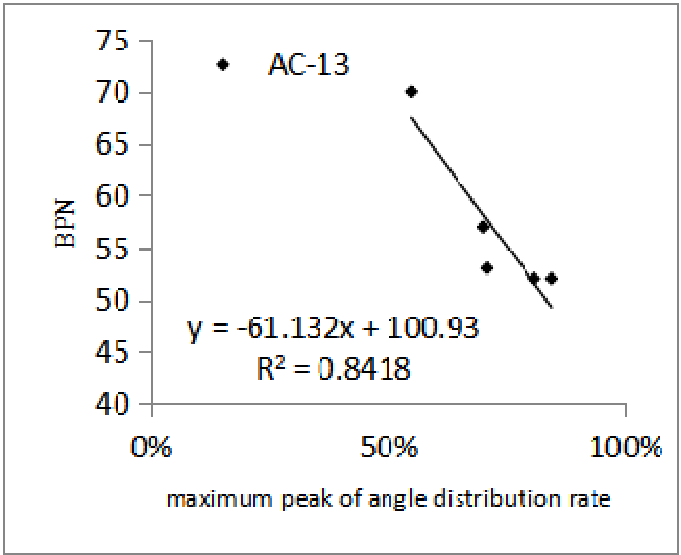

(c)

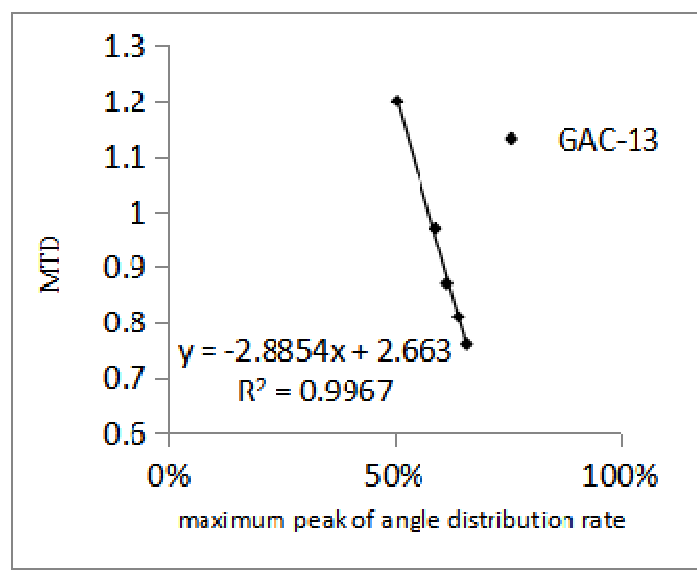

(b)

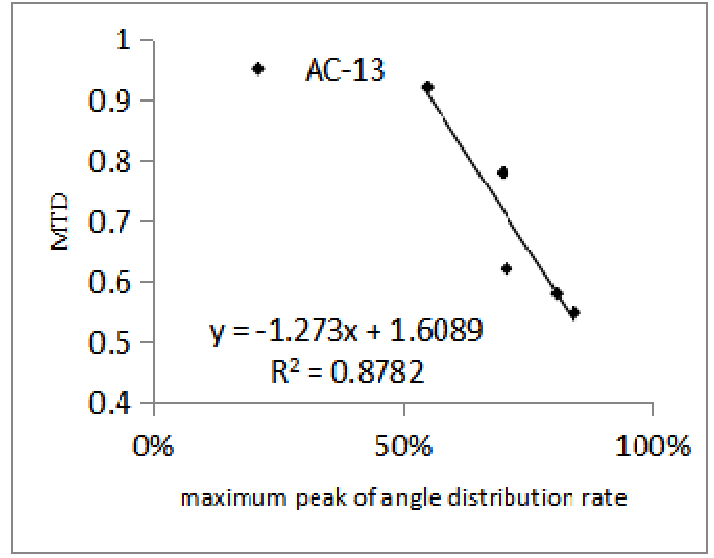

(d)

Figure 12. Correlation analysis of GAC-13 and AC-13.

\subsection{Designing anti-sliding durable type of asphalt mixture based on the maximum peak of angular distribution rate}

The anti-sliding performance and durability of the anti-skid layer of asphalt pavement (layer above) is directly related to the safety of the road traffic. But nowadays, in the asphalt mixture design phase, there has no special anti-sliding design and anti-sliding performance verification. This paper used the self-developed laser detector and acceleration load knead machine, and treated maximum peak of angular distribution rate as the anti-sliding performance evaluation index in the asphalt mixture design phase, so as to make up the absence of anti-sliding design and validation in anti-sliding layer asphalt mixture design phase nowadays.

Maximum peak of angular distribution rate in different stages of different gradations is shown in Table 5 6.

We can see from table 5: (1) The maximum peak of angular distribution rate of SMA is much lower than that of $\mathrm{AC}$, no matter in the beginning or after 8 hours kneading time. It means that SMA has better anti-sliding performance and durability; (2) Comparing the increasing trend of maximum peak of angular distribution rate of SMA rutting plates of 7 different gradations, we can see that before rubbing phase, the maximum peak of angular distribution rate of number $1 \sim 3$ rutting plate is gradually increasing, while the increase of that of number $5 \sim 7$ rutting plate is not obvious. The pass percentage of $9.5 \mathrm{~mm}$ and $4.75 \mathrm{~mm}$ sieve holes in number $1 \sim 3$ rutting plate is large, that is to say the 
aggregate is a bit over-fine. With the increase of coarse aggregate in 1 4 gradation, the initial and final maximum peak angular distribution rate have decreased, and the trend is obvious. Meanwhile, the maximum peak angular distribution rate of 1 4 gradation after 8 hours kneading time is still decreasing. In summary, the maximum peak of angular distribution rate of $1 \sim 4$ gradation is decreasing with the enlargement of aggregate gradation, and the later phase of kneading test also has the same rule. So we can say the anti-sliding performance and durability of SMA 1 4 has increased with the enlargement of aggregate gradation while through the data statistics of 5 7 plate, we can see that even if the aggregate gradation has the same enlargement, the change of maximum peak of angular distribution rate is not obvious, and comparing with number 4, it has a increase trend which still exists after 8 hours kneading time. Therefore, increasing the mixture ratio of number $5 \sim 7$ has no substantial contribution to anti-sliding performance and durability of the pavement, which, instead, would damage the anti-sliding performance and durability and make them reduce in the later period; (3) Therefore, we suggest using SMA-4 as the gradation of the anti-sliding layer of the pavement, and making proper adjustments of the pass percentage of $9.5 \mathrm{~mm}$ and $4.75 \mathrm{~mm}$ sieve holes for the pavement engineering, under the insurance of the effective anti-sliding performance and durability.

Table 5. Statistical table of maximum peak of angular distribution rate in different stages of SMA-13.

\begin{tabular}{l|c|c|c|c|c|c|c|c}
\hline & \multicolumn{7}{|c}{ Grading Type } & \multicolumn{1}{c}{} \\
\cline { 2 - 9 } & SMA-1 & SMA-2 & SMA-3 & SMA-4 & SMA-5 & SMA-6 & SMA-7 & AC \\
\hline 0(h) & $40.8 \%$ & $41.6 \%$ & $42.8 \%$ & $41.5 \%$ & $42.4 \%$ & $42.9 \%$ & $42.7 \%$ & $54.9 \%$ \\
\hline $\mathbf{2}$ (h) & $49.5 \%$ & $48.5 \%$ & $49.4 \%$ & $49.1 \%$ & $50.5 \%$ & $51.5 \%$ & $51.2 \%$ & $70.0 \%$ \\
\hline $\mathbf{4}$ (h) & $53.2 \%$ & $52.9 \%$ & $52.6 \%$ & $51.5 \%$ & $52.2 \%$ & $53.2 \%$ & $54.3 \%$ & $70.8 \%$ \\
\hline $\mathbf{6}$ (h) & $55.5 \%$ & $54.5 \%$ & $54.9 \%$ & $53.2 \%$ & $54.0 \%$ & $54.5 \%$ & $55.2 \%$ & $80.8 \%$ \\
\hline $\mathbf{8}$ (h) & $58.1 \%$ & $57.9 \%$ & $56.5 \%$ & $55.8 \%$ & $56.1 \%$ & $56.8 \%$ & $57.2 \%$ & $84.4 \%$ \\
\hline
\end{tabular}

Table 6. Statistic table of the maximum peak of angular distribution rate of GAC-13 in different stages.

\begin{tabular}{l|c|c|c|c|c|c|c|c}
\hline & \multicolumn{7}{|c}{ Grading Type } \\
\cline { 2 - 8 } & GAC-1 & GAC-2 & GAC-3 & GAC-4 & GAC-5 & GAC-6 & GAC-7 & AC \\
\hline 0 (h) & $48.9 \%$ & $49.6 \%$ & $50.8 \%$ & $50.6 \%$ & $51.3 \%$ & $51.5 \%$ & $51.7 \%$ & $54.9 \%$ \\
\hline $\mathbf{2}$ (h) & $58.3 \%$ & $59.2 \%$ & $60.3 \%$ & $59.2 \%$ & $58.5 \%$ & $59.3 \%$ & $60.1 \%$ & $70.0 \%$ \\
\hline $\mathbf{4}$ (h) & $61.1 \%$ & $61.9 \%$ & $62.6 \%$ & $61.7 \%$ & $62.5 \%$ & $61.0 \%$ & $62.0 \%$ & $70.8 \%$ \\
\hline $\mathbf{6}$ (h) & $65.1 \%$ & $65.6 \%$ & $65.4 \%$ & $64.2 \%$ & $65.0 \%$ & $66.4 \%$ & $65.5 \%$ & $80.8 \%$ \\
\hline $\mathbf{8}$ (h) & $67.9 \%$ & $67.1 \%$ & $66.5 \%$ & $66.0 \%$ & $66.4 \%$ & $67.1 \%$ & $67.2 \%$ & $84.4 \%$ \\
\hline
\end{tabular}

We can see from Table 6: (1) The maximum peak of angular distribution rate of GAC is much lower than that of AC, no matter in the beginning or after 8 hours kneading time. It means that GAC has better anti-sliding performance and durability as a anti-skid layer; (2) Comparing the increasing trend of the maximum peak of angular distribution rate of 7 different gradations of GAC rutting plates, we can see that before rubbing phase, the maximum peak of angular distribution rate of number 1 3 rutting plate is gradually increasing, while the increase of that of number 4 7 rutting plate is not obvious. The pass percentage of $9.5 \mathrm{~mm}$ and $4.75 \mathrm{~mm}$ sieve holes in number $1 \sim 3$ rutting plate is large, that is to say the aggregate is a bit over-fine. With the increase of coarse aggregate in 1 4 gradation, the initial and final maximum peak of angular distribution rate have decreased, and the trend is obvious. Meanwhile, the maximum peak of angular distribution rate of 1 4 gradation after 8 hours kneading time is still decreasing. In summary, the maximum peak of angular distribution rate of 1 4 gradation is decreasing with the enlargement of aggregate gradation, and this rule also works with the later phase of kneading test. So we can say the anti-sliding performance and durability of GAC 1 4 has increased with the enlargement of aggregate gradation while through the data statistics of number 5 7 mixture, we can see that even if the aggregate gradation has the same enlargement, the change of maximum peak of angular distribution rate is not obvious, and comparing with number 4 , it has a 
increase trend which still exists after 8 hours kneading time. Therefore, increasing the mixture ratio of number 5 7 has no substantial contribution to anti-sliding performance and durability of the pavement, which, instead, would damage the anti-sliding performance and durability and make them reduce in the later period; (3) Therefore, we suggest using GAC-4 as the gradation of the anti-sliding layer of the pavement, and making proper adjustments of the pass percentage of $9.5 \mathrm{~mm}$ and $4.75 \mathrm{~mm}$ sieve holes for the pavement engineering, under the insurance of the effective anti-sliding performance and durability; (4) In terms of GAC-13 and SMA-13 pavement, the pass percentage of $9.75 \mathrm{~mm}$ sieve holes is changed in number $1 \sim 3$ mixture; the pass percentage of $4.75 \mathrm{~mm}$ sieve holes is changed in number 3 5 mixture and that of $9.75 \mathrm{~mm}$ sieve holes is changed in number $5 \sim 7$ mixture. In this way, we continuously thickening the mixture gradation, and find that anti-sliding performance and durability of the pavement changes significantly when the pass percentage $4.75 \mathrm{~mm}$ sieve holes is changed, which means that reasonably controlling the pass percentage of $4.75 \mathrm{~mm}$ sieve holes is the key to the anti-sliding performance design of asphalt mixture; (5) To sum up, the anti-sliding performance: SMA $>$ GAC $>$ AC, and SMA-4 and GAC-4 have excellent anti-sliding performance and durability, which is commended as anti-sliding layer of the pavement. SMA-4 is more preferable when the conditions permit.

\section{Conclusion}

(1) Based on laser contour detection technology and self-developed accelerated rub tester, and a lot of data analysis, this paper propose that the maximum peak angular distribution rate could represent the anti-sliding performance and durability of asphalt pavement. Meantime, the correlation analysis between BPN and texture depth shows that they have a significant relation. It is sufficiently accurate to use maximum peak angular distribution rate to evaluate the anti-sliding performance and durability of asphalt pavement. It remedies the defect that the measure of the BPN and texture depth has being affected by uncontrollable factors like human and external environment.

(2) We use the maximum peak angular distribution rate to represent the skid resistance performance and durability and design different graduations of anti-sliding pavement layer, especially changing the pass rate of two gradations of sieve holes of $9.5 \mathrm{~mm}$ and $4.75 \mathrm{~mm}$. A conclusion can be made that the on the aspect of anti-sliding performance: SMA $>$ GAC $>A C$, and SMA-4 and GAC-4 have excellent anti-sliding performance and durability, which is recommended as anti-sliding layer of the pavement. SMA-4 is more preferable when the conditions permit. Meanwhile, with proper adjustments, two gradations of sieve holes of $9.5 \mathrm{~mm}$ and $4.75 \mathrm{~mm}$ are used in the pavement engineering.

(3) According to the laser contour detection test and method for evaluating anti-sliding performance of the pavement, we form a set of laboratory system, which is able to design and verify the anti-sliding performance and durability of the asphalt mixture in the design stage, which provides guidance for the engineering application.

\section{References}

1. J. W. Hall, K. L. Smith, and L. Titus-Glover, NCHRP: guide for pavement friction, Washington DC: Transportation Research Record (2009)

2. Guidelines for the management of road surface skid resistance, Sydney: Austroads Incorporated (2005)

3. E. Farber, M. S. Janoff, S. Cristinzio, J. G. Blubaugh, W. Reisener, and W. Dunning, Determining pavement skid-resistance requirements at intersections and braking sites, Washington DC: National Cooperative Highway Research Program, 154 (2004)

4. T. R. Nesbitt and D. J. Barron, Prediction of driving traction performance on snow, SAE Technical Paper (1980)

5. T. R. King and F. E. Matyja, Tread design effect on winter traction, PA: Society of Automotive Engineers (1981) 
6. P. Cao and X. P. Yan, Theory analyses of effect of topography on skid resistance of asphalt pavements, Tribology, 29 (4), 306-310 (2009)

7. Z. L. Zhao, Z. Q. Zhang, and C. S. Hu, Influence of gradation on anti-skidding performance of asphalt pavement, Journal of Chang' an University, 25 (1), 6-9 (2005)

8. Y. Zhang, Research of gradation optimization on anti-sliding layer of asphalt pavement, Chang Chun: Ji Lin University (2007)

9. X. S. Du, Study of pavement anti-skid performance based on tire and road interaction, Harbin: Harbin Institute of Technology (2012)

10. A. Q. Zhang and Z. Y. Ao, Tire contact pressure distribution and test methods, China Rubber Industry, 48 (6), 368-374 (2001)

11. M. D. Beer, L. Kannemeyer, and C. Fisher, Towards improved mechanistic design of thin asphalt layer surfacings base on actual tyre/ pavement contact stress-in motion(SIM) data in south Africa, Proceedings of 7th Conference on asphalt pavements for Southern Africa (1999)

12. L. W. Shi, Based on the functional properties of micromechanics asphalt research, Guangzhou: South China University of Technology (2014) 